\title{
PRODUCTO, DESEMPLEO Y LA LEY DE OKUN EN LA REPÚBLICA DOMINICANA
}

Ouput, unemployment and Okun's law in the Dominican Republic

\author{
José R. Sánchez-Fung* \\ Universidad de Nottingham, Ningbo, China, y \\ Universidad de Londres, ILAS, Reino Unido
}

Resumen: El artículo investiga la Ley de Okun para la República Dominicana durante la segunda mitad del siglo XX y la primera década del XXI. El análisis econométrico estima un coeficiente de Okun implicando que, en promedio, cada 1 porciento de crecimiento en el producto real genera una reducción de 0.5 puntos porcentuales en el nivel del desempleo. Pero las estimaciones recursivas revelan que el coeficiente de Okun ha caído en los últimos cuarenta años alcanzando -0.88 en 1966 y registrando -0.5 en 2013. El desplome del coeficiente de Okun y la inercia en el número de trabajadores clasificados fuera de la oferta de trabajo pueden ayudar en la explicación de episodios de "crecimiento sin empleo" observados en la República Dominicana. El análisis también revela que el crecimiento anual promedio del producto real necesario para mantener estable la tasa de desempleo es $4.5 \%$.

José R. Sánchez-Fung, University of Nottingham, Department of Quantitative and Applied Economics, Business School, 199 Taikang.

E-mail: JoseR.Sanchez-Fung@nottingham.edu.cn. 
Palabras claves: Ley de Okun; producto; desempleo; República Dominicana.

JEL clasificación: E24; E32.

Reconocimientos: El artículo fue preparado para el taller INTECUniversidad de Londres The Dominican Republic's Economy celebrado en el Instituto de Estudios Latinoamericanos, Universidad de Londres, Reino Unido, el 7 de noviembre de 2014. La Escuela de Negocios de la Universidad de Nottingham en Ningbo, China, otorgó financiamiento para participar en el evento. El taller contó con el respaldo de la revista Ciencia y Sociedad del INTEC, Santo Domingo, República Dominicana. Agradezco los comentarios y sugerencias de Julio G. Andújar-Scheker, Jaime Aristy-Escuder, Susan Pozo y Amelia U. Santos-Paulino sobre versiones anteriores del estudio. Cualquier error u omisión es responsabilidad del autor.

Abstract: The paper investigates Okun's Law for the Dominican Republic during the second part of the twentieth century and the first decade of the twenty first. The econometric modelling estimates an Okun coefficient implying that, on average, for every 1 percent growth in real output the unemployment rate decreases 0.5 percentage points. But recursive modelling reveals that Okun's coefficient has being falling during the last forty years from -0.88 in 1966 to -0.5 in 2013 . The drop in the magnitude of Okun's coefficient and the inertia in the number of workers registered out of the labour supply could help in illuminating episodes of "jobless growth" observed for the Dominican Republic. The analysis also reveals that the implicit average rate of real output growth consistent with stable unemployment is $4.5 \%$ per annum.

Keywords: Okun's Law; output; unemployment; Dominican Republic.

JEL classification: E24; E32. 
Acknowledgements: The paper was prepared for the INTECUniversity of London workshop The Dominican Republic's Economy held at the Institute for Latin American Studies, University of London, UK, on 7 November 2014. I thank the University of Nottingham Business School, Ningbo, China for a conference grant to attend the event. The workshop was co-sponsored by the journal Ciencia y Sociedad published by INTEC in Santo Domingo, Dominican Republic. I thank Julio G. Andújar-Scheker, Jaime Aristy-Escuder, Susan Pozo and Amelia U. Santos-Paulino for comments and suggestions on previous drafts. Any remaining errors are the author's responsibility

\section{Introducción}

Entender el ciclo de negocios es importante porque las fluctuaciones adversas en el producto y el desempleo pueden perjudicar el bienestar de los agentes económicos. Por ejemplo, las decisiones del Banco Central sobre la postura de la política monetaria dependen del impacto esperado de un cambio en el producto sobre el mercado de trabajo. Como ilustración, la conocida Regla de Taylor, usada alrededor del mundo para informar las decisiones de política monetaria, implícitamente asume que las desviaciones del producto de su potencial involucran la existencia de desviaciones del desempleo de su tasa natural.

La Ley de Okun surge de estudios empíricos mostrando la existencia de una relación negativa entre el producto y el desempleo (Okun, 1962; Ball, Leigh y Loungani, 2013; Daly y otros, 2014). Pero la conclusión de la investigación original de Arthur Okun y de estudios subsecuentes sobre los Estados Unidos, implicando una relación 2:1 entre el producto y el desempleo, y que es parte de los libros de texto sobre macroeconomía, no puede ser empleada 
de forma mecánica para la toma de decisiones en otros países. Por lo tanto el objetivo de la presente investigación es contestar las siguientes preguntas: ¿Existe una relación empírica entre el producto y el desempleo en la República Dominicana? ¿En dicho caso, es el coeficiente de Okun estable? ¿Y qué podemos aprender de la evolución histórica del coeficiente de Okun con relación al desenvolvimiento de la macroeconomía?

La literatura contiene escasos trabajos sobre la Ley de Okun aplicada al entendimiento de países en vías de desarrollo. García Obregón (2013) analiza la relación entre el producto y el desempleo en la República Dominicana. El análisis emplea datos trimestrales para el período 2000-2011, de manera tal que las estadísticas restringen el potencial de entender la relevancia o no de la Ley de Okun ante el cambio institucional y el desarrollo de la economía en general. Una posible razón por lo cual no existen trabajos sobre la Ley de Okun para la República Dominicana para un período suficientemente extendido es la carencia de datos agregados sobre el mercado laboral (Sánchez-Fung, 2000).

La presente investigación contribuye adoptando una visión de largo plazo que implica investigar medio siglo de series estadísticas sobre el producto y el desempleo empleando técnicas econométricas. El período analizado incluye la transición del país de una dictadura a la democracia durante la década de 1960; la experimentación con una estrategia de desarrollo económico basada en la sustitución de importaciones y la promoción de la industria local, y choques adversos afectando los precios del petróleo a nivel internacional durante la década del setenta; la crisis de la deuda y la primera devaluación del peso dominicano en los años ochenta; la implementación de un proceso extenso de reformas estructurales a principios de la década del noventa; y dos crisis económicas significativas en 1990 y en 2003-2004. En vista de la compleja dinámica de la economía de la República Dominicana, durante el período la investigación se aplican técnicas econométricas recursivas para examinar la evolución del comportamiento de los coeficientes de interés. 
El resto de la investigación procede de la siguiente manera. La sección 2 explica aspectos analíticos de relevancia para entender la Ley de Okun. La sección 3 discute las tendencias en las estadísticas del producto real y el desempleo en la República Dominicana durante la segunda mitad del siglo XX y la primera década del XXI. La sección 4 implementa los análisis econométricos, la sección 5 discute las implicaciones de los resultados de la modelación empírica para el entendimiento de la evolución del mercado de trabajo y de la macroeconomía en la República Dominicana. La sección 6 concluye la investigación.

\section{Aspectos analíticos de la Ley de Okun}

La Ley de Okun plantea la base para investigar la existencia de una relación empírica entre el desempleo y el producto a nivel agregado. Expresando la ecuación más básica tenemos que

$$
U=\alpha+\partial Y
$$

En la ecuación (1) $Y$ es el producto real y $U$ la tasa de desempleo en la economía. El parámetro $\delta$ es conocido como el coeficiente de Okun. Existen varias versiones de la ecuación 1, como señala Knotek (2007), y la primera ecuación originalmente planteada por Okun es

$$
\Delta U=\alpha+\delta \Delta Y
$$

La ecuación 2 muestra la relación entre el cambio en el producto y el desempleo -donde el operador $\Delta$ indica el cambio de una variable-. La interpretación de la ecuación 2 puede ser muy intuitiva; por ejemplo, si $\delta=0$ entonces la tasa de crecimiento del desempleo es $\alpha$. Una ventaja de la ecuación 2 es que no es necesario calcular variables como el producto potencial o la tasa natural de desempleo, lo cual introduce complicaciones en la modelación econométrica. 
Sin embargo, otra versión considerada por Okun plantea el uso de variables no observadas, calculando brechas, y tenemos que

$$
U=\alpha+\delta\left(Y-Y^{*}\right)
$$

La ecuación (3) contiene variables definidas anteriormente para el desempleo y el producto, e introduce la brecha entre el producto actual y el producto potencial $\left(Y^{*}\right)$. La ecuación 3 implica que si el producto se encuentra en su nivel potencial $\left(Y-Y^{*}=0\right)$ la tasa natural de desempleo es $\alpha$. Esto implica que si sustraemos $\alpha$ de ambos lados de la ecuación 3 tenemos una relación entre la brecha del producto respecto al potencial $\left(Y-Y^{*}\right)$ y del desempleo con relación a la tasa natural de desempleo $(U-\alpha)$. El coeficiente de Okun $\delta$ debe ser negativo, implicando que si el producto real se encuentra por encima del potencial $\left(Y>Y^{*}\right)$ el desempleo disminuye $(U \downarrow)$ y vice-versa.

El carácter estático de las relaciones planteadas anteriormente es criticado por Okun y subsecuentemente por otros investigadores (p. ej., Gordon, 1984). La crítica conduce a plantear versiones de las ecuaciones expresadas en tasas de crecimiento y considerando rezagos del desempleo y del producto

$$
\Delta U_{t}=\alpha+\sum_{i=1}^{n} \beta_{i} \Delta U_{t-i}+\sum_{i=0}^{n} \delta_{i} \Delta Y_{t-i} .
$$

La ecuación 4 introduce el operador $\sum$ indicando la sumatoria desde $i$ hasta $n$ de los coeficientes sobre las variables rezagadas.

Prachowny (1993) explica que entre las críticas a la ley de Okun se encuentra el hecho de que existen factores detrás de las variaciones en el producto que deben ser considerados explícitamente para explicar el desempleo: horas trabajadas, productividad y fuerza laboral. Esto conduce a derivar versiones de la Ley de Okun combinado la versión basada en brechas con otros elementos que forman parte de una función de producción. La ecuación de interés derivada por Prachowny, en la cual el producto es la variable dependiente, es: 


$$
Y-Y^{*}=\varnothing\left(C-C^{*}\right)+\tau\left(L-L^{*}\right)+\theta\left(H-H^{*}\right)+\gamma\left(U-U^{*}\right)
$$

En la ecuación 5 la brecha del producto $\left(Y-Y^{*}\right)$ y la brecha del desempleo $\left(U-U^{*}\right)$ son conocidas. Pero la nueva formulación incluye variables, derivadas de una función de producción, para capturar la utilización del capital $\left(C-C^{*}\right)$, la brecha en la oferta laboral $\left(L-L^{*}\right)$ y las horas trabajadas $\left(H-H^{*}\right)$. Los parámetros $\emptyset, \tau, \theta, \gamma$ deben ser determinados empíricamente. Los asteriscos indican valores de largo plazo. Para obtener la ecuación 5 Prachowny asume que el factor tecnológico y el stock de capital, presentes en la función de producción original, se encuentran en sus valores de equilibrio. La ecuación 5 podría ser de gran utilidad para entender la relación entre el producto y el desempleo. Sin embargo, para una economía como la de República Dominicana no existen series confiables para aproximar las horas trabajadas y la utilización del capital y esa realidad imposibilita el empleo juicioso de una ecuación como 5.

\section{Producto real y desempleo en la República Dominicana}

Los datos sobre el producto real y la tasa de desempleo (ampliada) para la República Dominicana abarcan el período 1960-2013. Las estadísticas son de frecuencia anual y las fuentes son el Banco Mundial para el producto real y el Banco Central de la República Dominicana para el desempleo. Las series del producto y el desempleo empleadas en esta sección son expresadas en logaritmos $(y, u)$ de manera tal que las diferencias son tasas de crecimiento:

$$
\Delta y_{t}=y_{t}-y_{t-1} \text { y } \Delta u_{t}=u_{t}-u_{t-1} \text {. }
$$

La figura N..$^{\circ} 1$ muestra la tasa de desempleo expresada en por ciento. La observación más elevada se registra en 1965 durante la Guerra Civil cuando el desempleo alcanza 35\%. El desempleo se estabiliza alrededor de $20 \%$ durante los 1970 y las siguientes 
observaciones más elevadas coinciden con las crisis financieras en los años 1985 (25\%) y 1990 (23\%). Durante los 1990 el desempleo disminuye considerablemente, fluctuando entre $20 \%$ y $13 \%$, probablemente como resultado del extenso proceso de reformas económicas implementado a principios de la década en el contexto del Consenso de Washington. ${ }^{1}$ Subsecuentemente la tendencia a la baja es interrumpida por la crisis bancaria de los años 2003-2004 y el desempleo registra 18\% en el año 2004 para luego retornar a los niveles observados a finales de los 1990.

\section{Figura N. ${ }^{\circ} 1$}

\section{Desempleo en la República Dominicana, porciento, 1960-2013}

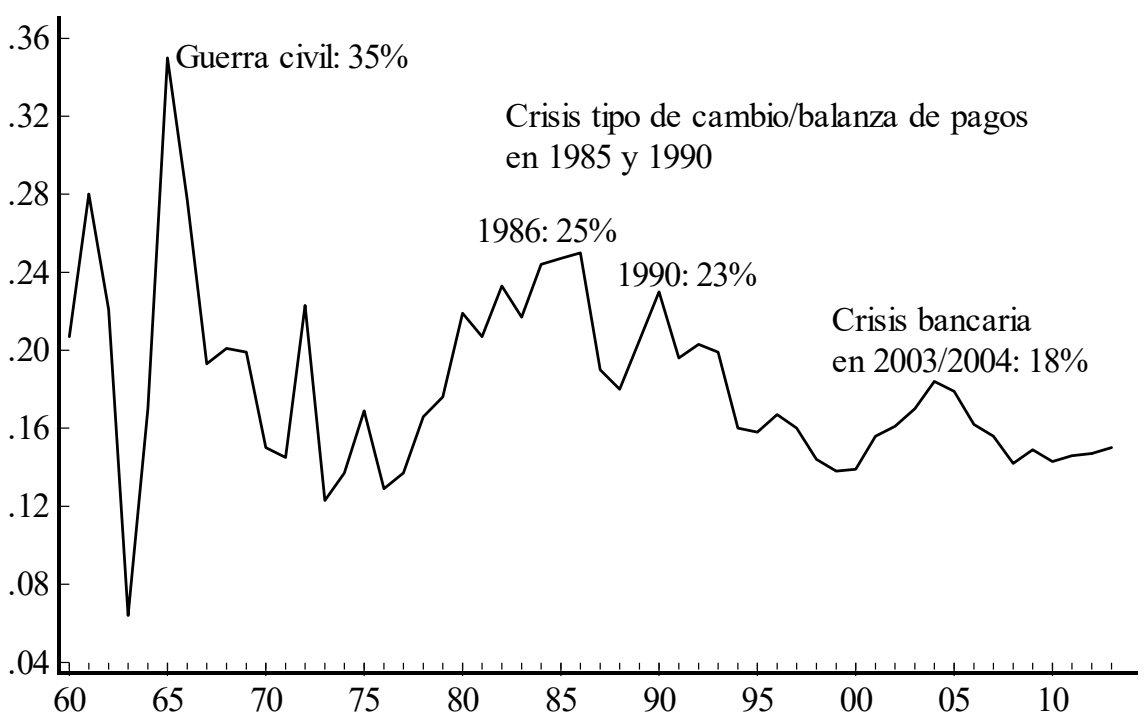

Fuente: Banco Central de la República Dominicana.

El crecimiento del producto real $(\Delta y)$ en la figura $\mathrm{N} .^{\circ} 2$ refleja las fluctuaciones en el mercado de trabajo. Las principales caídas en el producto real corresponden a la Guerra civil en 1965 y a las crisis económicas de 1985, 1990 y 2003. Y las observaciones más

1 Ver Sánchez-Fung (2005). 
elevadas corresponden al período de sustitución de importaciones y precios favorables para los bienes primarios de exportación, registrando $16.7 \%$ en 1970 y $12.1 \%$ en $1973 .{ }^{2}$ Los valores más elevados subsecuentemente son observados alrededor de dos años posteriores a la implementación de acuerdos de estabilización con el Fondo Monetario Internacional (FMI): 1987 (9.6\%), 1992 (10\%) y 2006 (10.1\%). El país firmó acuerdos con el FMI en 1985, 1990 y 2003-2004.

\section{Figura N. ${ }^{\circ} 2$}

Crecimiento del producto real en la República Dominicana Diferencia del logaritmo, 1961-2013

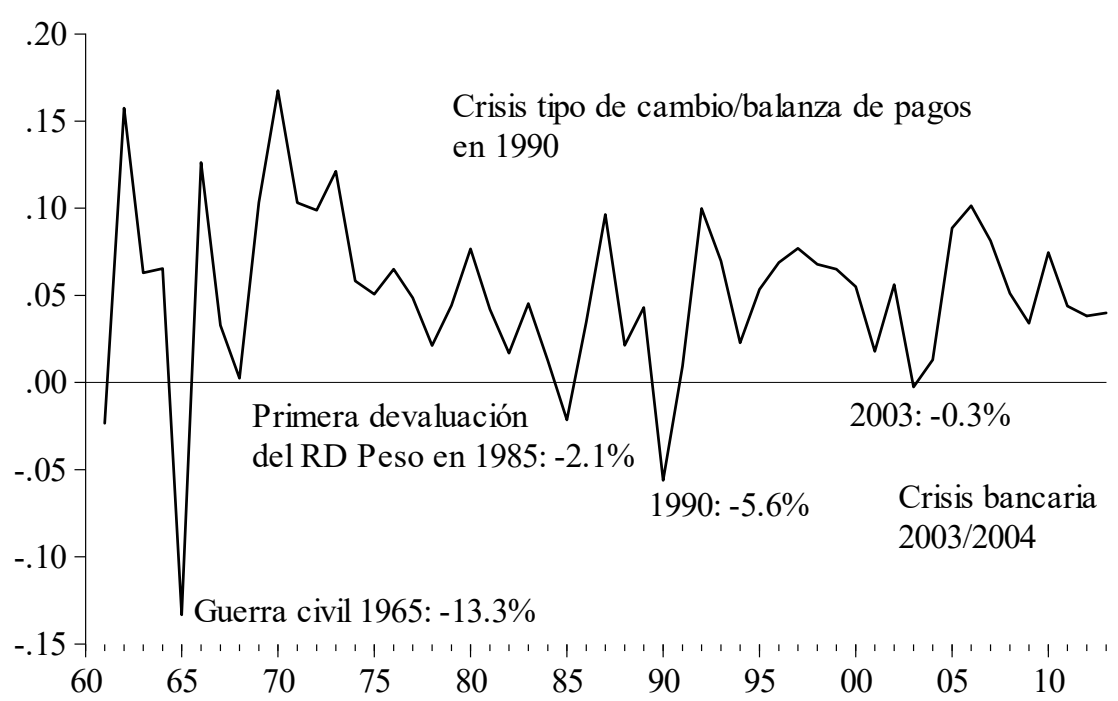

Fuente: Banco Mundial.

La figura $\mathrm{N} .^{\circ} 3$ contiene evidencia visual sobre la relación entre el crecimiento del producto real $(\Delta y)$ y el crecimiento de la tasa de desempleo $(\Delta u)$. Para facilitar la interpretación de las series la figura emplea una elipse y una línea de regresión. La línea de regresión tiene pendiente negativa, lo cual es evidencia informal de la

2 Ver Pozo, Sánchez-Fung y Santos-Paulino (2013). 
existencia de una relación como la postulada por la Ley de Okun. Es importante destacar que las únicas observaciones que se encuentran fuera de la elipse son las correspondientes a los años 1963, 1964 y 1965. Y este es el período de transición desde una dictadura de 30 años de duración hacia la democracia empezando en 1961. La figura $\mathrm{N} .{ }^{\circ} 3$ muestra evidencia informal implicando que una tasa de desempleo estable es compatible con un crecimiento real de la economía de alrededor de $5 \%$ por año -las estimaciones econométricas presentadas más adelante corroboran las cifras.

\section{Figura N. ${ }^{\circ} 3$}

Crecimiento del producto real y del desempleo

Diferencia de logaritmos, con elipse y línea de regresión, 1961-2013

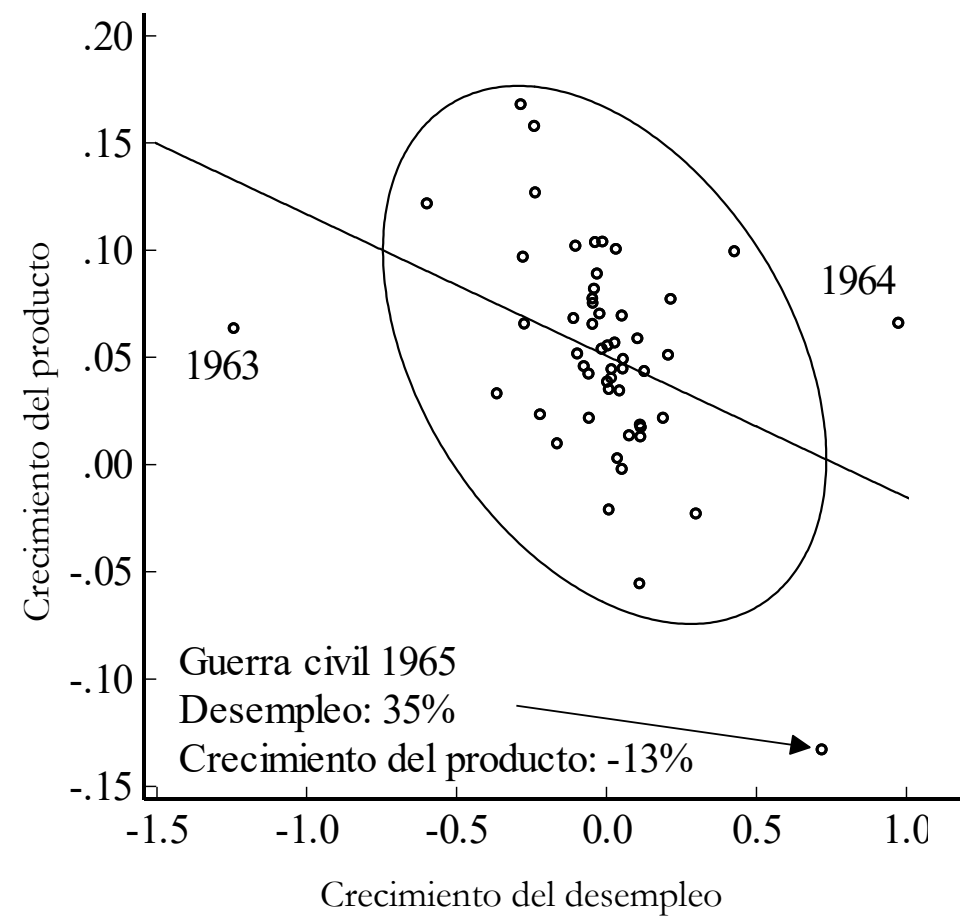

Fuentes: Banco Central de la República Dominicana, Banco Mundial y estimaciones del autor. 
La relación entre el producto real y el desempleo es similar si empleamos brechas en lugar de tasas de crecimiento. La brecha del producto real $y_{t}-y_{t}^{*}$ es calculada como la diferencia entre el logaritmo del producto real $(y)$ y logaritmo del producto potencial $\left(y^{*}\right)$ calculado empleando el filtro de Hodrick-Prescott con un valor de 6.5. La tasa natural de desempleo $\left(u^{*}\right)$ es calculada pasando la tasa de desempleo $(u)$ por el mismo filtro y la variable resultante es empleada para obtener la brecha del desempleo $u_{t}-u_{t}^{*}$.

Figura N. ${ }^{\circ} 4$

Brechas del producto real y del desempleo

Diferencia de logaritmos, con elipse y línea de regresión, 1961-2013

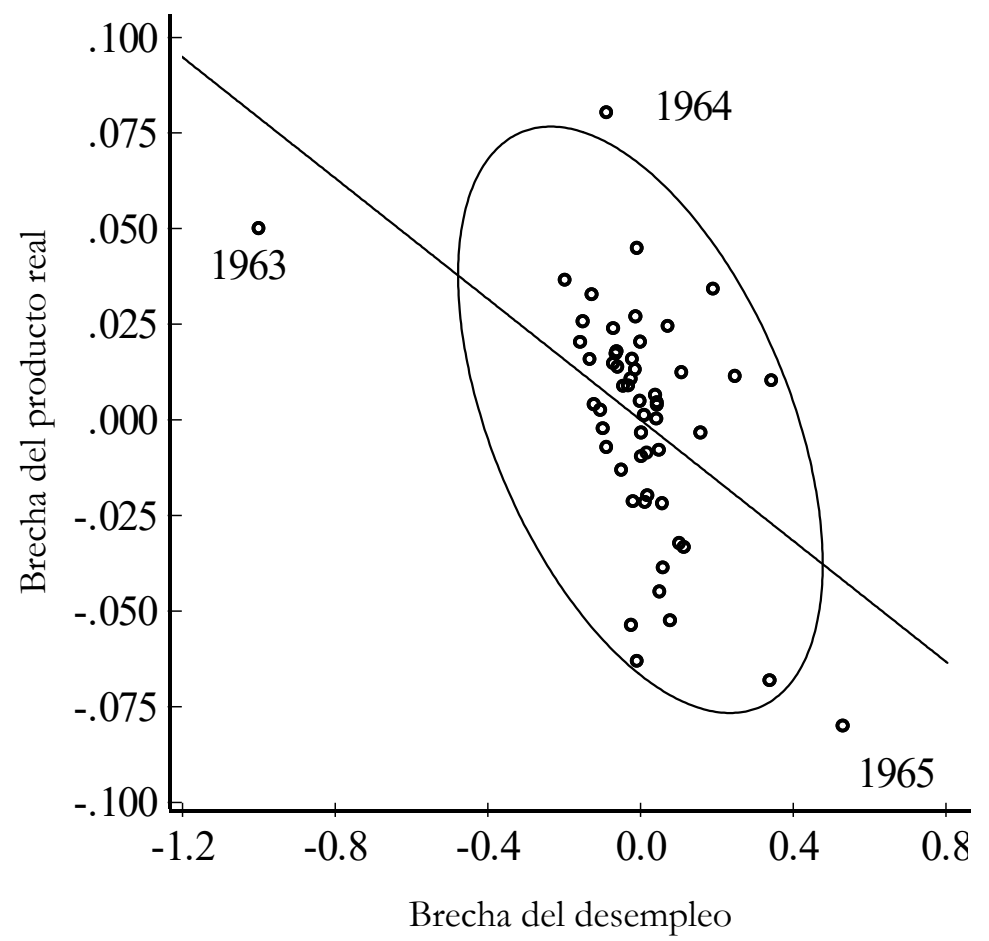

Fuentes: Banco Central de la República Dominicana, Banco Mundial y estimaciones del autor. 
La figura N. ${ }^{\circ} 4$ contiene las dos brechas y muestra que, al igual que la figura $\mathrm{N} .^{\circ} 3$, las únicas observaciones que se encuentran fuera de la elipse son las correspondientes a los años 1963, 1964 y 1965. Los análisis econométricos desarrollados en la siguiente sección deben arrojar más luz sobre la relevancia de la Ley de Okun para la República Dominicana.

\section{Modelación econométrica de la relación entre el desempleo y el producto real}

El análisis econométrico considera tres especificaciones alternativas para analizar la Ley de Okun en la República Dominicana. Dos modelos utilizan como variable dependiente la brecha del nivel del desempleo respecto a la tasa natural $\left(U_{t}-U_{t}^{*}\right)$ y el tercer modelo emplea la variación del nivel del desempleo $\left(U_{t}-U_{t-1}\right)$. En el lado derecho de las ecuaciones el producto entra en la primera especificación expresado como la brecha entre el logaritmo del producto actual y el potencial $\left(y_{t}-y_{t}^{*}\right)$ y en las dos siguientes ecuaciones expresado como la variación de su logaritmo $\left(y_{t}-y_{t-1}\right)$. Según lo expresado anteriormente, el valor natural y el potencial para el desempleo y el producto son computados aplicando el filtro de Hodrick-Prescott con un valor de 6.5. Los modelos son estimados empleando la técnica de mínimos cuadrados ordinarios (MCOs) y datos anuales para el período 1962-2013. La tabla N. ${ }^{\circ} 1$ reporta los coeficientes estimados para las ecuaciones de Okun junto con errores estándar consistentes con autocorrelación y heterocedasticidad.

Las primeras dos columnas en la tabla N. ${ }^{\circ} 1$ reportan los resultados para el modelo con la brecha del desempleo $\left(U_{t}-U_{t}^{*}\right)$ como variable dependiente y la brecha del producto $\left(y_{t}-y_{t}^{*}\right)$ en el lado derecho de la ecuación. La investigación estima la ecuación 6 reportada en la tabla $\mathrm{N} .^{\circ} 1$ y muestra que los rezagos de las variables no son estadísticamente significativos. El modelo es reducido y la ecuación 7 muestra que la constante es cero y estadísticamente 
no significativa; el coeficiente de Okun es significativo al 5\%. Es importante reiterar que el uso de modelos con brechas tiene la debilidad de depender sobre variables no observadas para el producto potencial y la tasa natural de desempleo, y esto puede introducir complicaciones en las estimaciones. Incluso para países avanzados, algunos investigadores optan por no emplear los modelos con brechas y prefieren emplear variaciones en el tiempo (p. ej., Knotek, 2007).

\section{Tabla N. ${ }^{\circ} 1$}

Estimaciones de la Ley de Okun para la República Dominicana (MCOs)

Versiones con brechas y con variaciones

de los logaritmos del producto real

1962-2013

\begin{tabular}{|l|c|c|c|c|}
\hline \multirow{2}{*}{$\begin{array}{c}\text { Lado derecho de 1a } \\
\text { ecuación }\end{array}$} & \multicolumn{3}{|c|}{ La variable dependiente es $\boldsymbol{U}_{\boldsymbol{t}}-\boldsymbol{U}_{\boldsymbol{t}}^{*}$} \\
\cline { 2 - 5 } & \multicolumn{2}{|c|}{$\begin{array}{r}\text { Brecha del producto } \\
y_{t}-y_{t}^{*}\end{array}$} & $\begin{array}{r}\text { Variación logaritmo producto } \\
y_{t}-y_{t-1}\end{array}$ \\
\cline { 2 - 5 } & Ecuación 6 & Ecuación 7 & Ecuación 8 & Ecuación 9 \\
\hline Constante & -0.00 & -0.00 & 0.02 & -0.01 \\
& $(0.00)$ & $(0.00)$ & $(0.01)$ & $(0.01)$ \\
\hline Rezago del desempleo & -0.01 & - & -0.02 & - \\
\hline Producto real & $(0.13)$ & $-0.11)$ & -0.21 \\
\hline Rezago del producto & -0.57 & -0.56 & -0.19 & $(0.11)^{*}$ \\
real & $(0.22)^{*}$ & $(0.22)^{*}$ & $(0.16)$ & - \\
\hline
\end{tabular}

\section{Notas:}

(1) $* *$ y $*$ indican coeficientes significativos al 1\% y 5\% de probabilidad.

(2) MCOs: mínimos cuadrados ordinarios.

(3) Los coeficientes estimados para las ecuaciones de Okun son reportados junto con errores estándar consistentes con auto correlación y heterocedasticidad entre paréntesis.

(4) Todas las variables empleadas en la modelación econométrica reportada en la tabla son estacionarias al $1 \%$ de probabilidad de acuerdo con la prueba DickeyFuller aumentada. Los valores de la prueba son: brecha del desempleo (-11.62), brecha del producto real (-5.61) y variación del logaritmo del producto real (-4.06). 
La tabla N. ${ }^{\circ} 1$ contiene la ecuación 8 presentando el modelo estimado con la brecha del desempleo $\left(U_{t}-U_{t}^{*}\right)$ como variable dependiente y la variación del producto $\left(y_{t}-y_{t-1}\right)$ como variable explicativa y considerando un rezago para cada variable; la única variable significativa es el rezago del producto. Reduciendo la ecuación 8 a un modelo estático no mejora los resultados de la ecuación 9 reportada en la última columna de la tabla N. ${ }^{\circ} 1$. Sin embargo, en ambas ecuaciones el coeficiente de Okun es negativo.

La modelación considera una tercera especificación de la Ley de Okun, la ecuación 2, usando la diferencia en el nivel de la tasa de desempleo $\left(U_{t}-U_{t-1}\right)$ y el cambio en el logaritmo del producto real $\left(y_{t}-y_{t-1}\right)$. Luego de considerar y descartar una especificación con rezagos, el análisis selecciona la siguiente ecuación computada usando el estimador de mínimos cuadrados ordinarios para el período 1962-2013.

$$
U_{t}-U_{t-1}=\underbrace{0.02}_{(0.009)}-\underbrace{0.50}_{(0.16)}\left(y_{t}-y_{t-1}\right) .
$$

En la ecuación 10 los valores entre paréntesis son errores estándar consistentes con auto correlación y heterocedasticidad e indican que la constante y el coeficiente de Okun son significativos al menos al 5\% de probabilidad. Los residuos de la ecuación 10 exhibidos en la figura $\mathrm{N} .^{\circ} 5$ muestran un patrón suave y claramente estacionario, lo cual es confirmado por la prueba de Dickey-Fuller aumentada de un modo significativo al 1\% (-5.17), y no reflejan la existencia de choques relevantes que perturben la relación de Okun. El valor del coeficiente de Okun es -0.5 , muy cercano al reportado por Ball, Leigh y Loungani (2013) para los Estados Unidos. La figura $\mathrm{N} .{ }^{\circ} 6$ contiene una elipse de confianza de 1\% de probabilidad que indica el valor negativo del coeficiente de Okun en todo el rango de la elipse. 
Figura N. ${ }^{\circ} 5$

Residuos de la ecuación de Okun número 10, 1962-2013

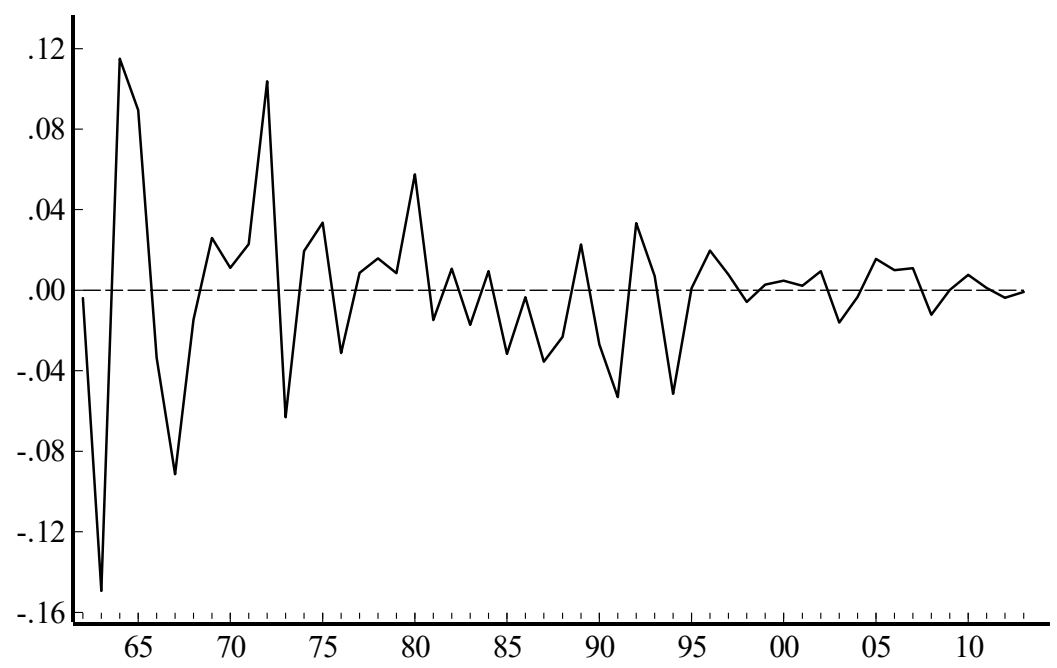

Figura N. ${ }^{\circ} 6$

Elipse de confianza de 1\% para el coeficiente de Okun, ecuación 10

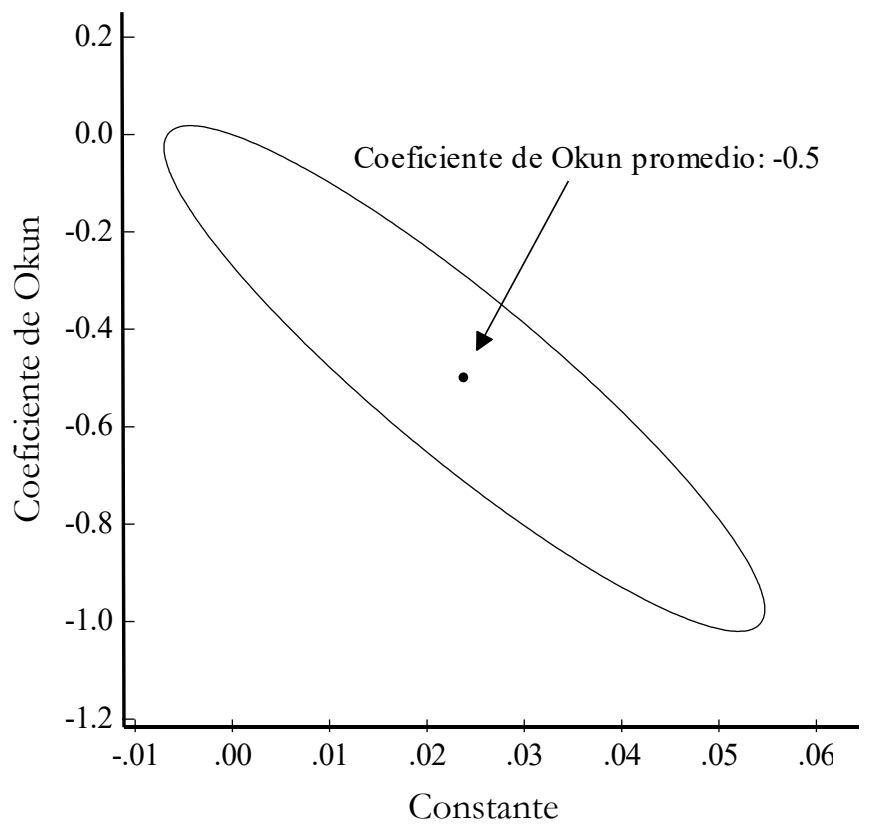

Ciencia y Sociedad 2015; 40(3): 613-637 627 
Usando los coeficientes de la ecuación 10 puede computarse el valor del crecimiento del producto real necesario para obtener una tasa de desempleo estable. La estimación del producto potencial es obtenida dividiendo la constante de la ecuación entre el coeficiente de Okun (con signo negativo). El cálculo muestra que $4.5 \%$ de crecimiento anual del producto real mantiene el desempleo estable durante la muestra estudiada. El número es cercano al estimado por García Obregón (2013) para la República Dominicana empleando datos trimestrales y una muestra que abarca la primera década del siglo XXI. García Obregón reporta una ecuación de cointegración similar a (10) con un coeficiente de Okun de -0.24 y constante de 0.01. Estos números implican que el crecimiento anual del producto real necesario para mantener una tasa de desempleo estable es $\{-(0.01 /-0.24)\}=0.0416$, o $4.16 \%$.

\section{ESTIMACIONES RECURSIVAS}

La economía de la República Dominicana ha experimentado cambios significativos desde la década de 1960 y es importante analizar la estabilidad del coeficiente de Okun. El análisis computa la ecuación 10 empleando métodos recursivos. El método recursivo estima el modelo econométrico para un período en el tiempo y actualiza las estimaciones hasta agotar el número total de observaciones estadísticas disponibles en la muestra. La técnica es útil para analizar el comportamiento del coeficiente estimado para una variable a medida que pasa el tiempo.

La figura N. ${ }^{\circ} 7$ contiene dos variables: el coeficiente de Okun y el valor implícito del crecimiento del producto real necesario para mantener la tasa de desempleo estable. La figura N. 7 muestra que el coeficiente de Okun registró -0.88 en 1966 y -0.50 en 2013. El valor más elevado para el coeficiente de Okun ocurre desde la década de 1960, alcanzando -0.88 en 1966. El coeficiente disminuye progresivamente y durante la década de 1970 fluctúa entre -0.70 y -0.63. La década de 1970 fue un período de crecimiento económico rápido como muestra la figura $\mathrm{N} .^{\circ} 1$. El crecimiento observado 
fue producto de varios factores, incluyendo la implementación de políticas de desarrollo económico basadas en una estrategia de sustitución de importaciones y precios internaciones favorables para los productos primarios de exportación. Y es el período que registra los valores más elevados para el producto potencial implícito, como muestra la figura $\mathrm{N} .^{\circ} 7$, fluctuando alrededor de $6 \%$.

\section{Figura N. ${ }^{\circ} 7$}

Coeficiente de Okun y valores del producto real consistentes con desempleo estable

Estimación recursiva de la ecuación 10, 1965-2013

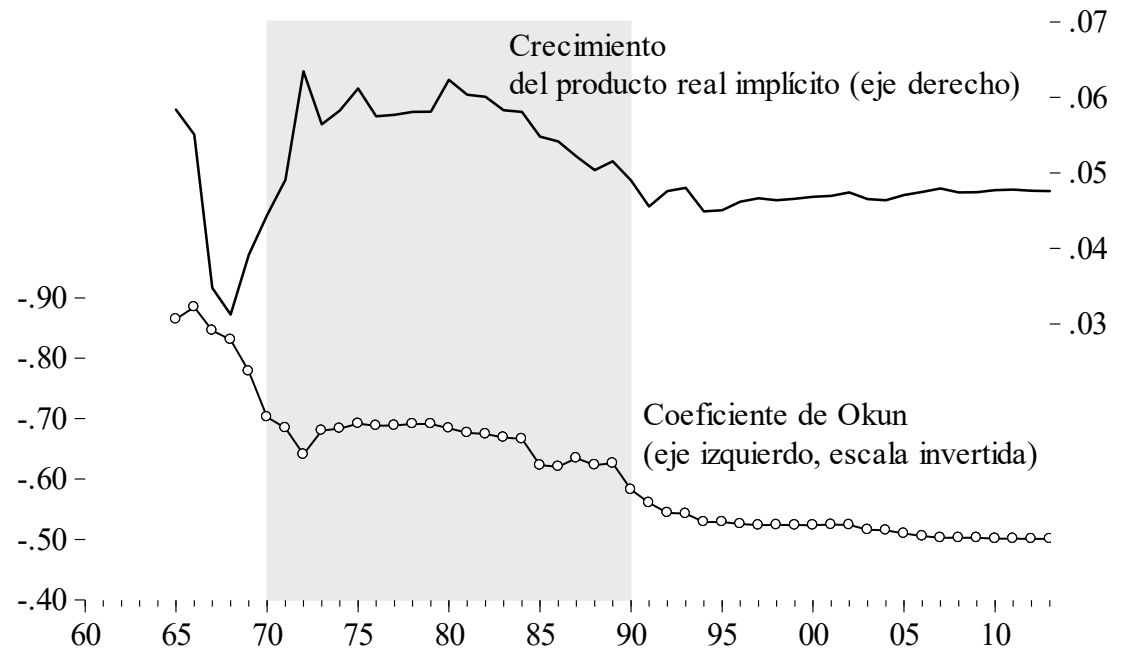

Los valores del coeficiente de Okun recursivo disminuyen durante toda la década de 1980 y terminan con un valor de -0.63 . Esta tendencia es paralela a la disminución del producto potencial que cae 1 punto porcentual desde $6.2 \%$ en 1980 hasta alcanzar 5.2\% en 1989. La década de 1980 es conocida como la década perdida para América Latina y la República Dominicana no es una excepción. El país ejecutó la primera devaluación del Peso dominicano en 1985 y firmó una serie de acuerdos con el FMI 
para estabilizar la economía. Finalmente, y no obstante las reformas mencionadas anteriormente inspiradas por el Consenso de W ashington, la figura N. ${ }^{\circ} 7$ muestra que tanto el coeficiente de Okun como el producto potencial caen durante los años de la década del noventa y los años a partir del 2000, en comparación con la zona sombreada en la figura correspondiente a la década del setenta y la década del ochenta (del pasado siglo XX). Durante las últimas dos décadas el producto real potencial se estabiliza alrededor de 4.5\% y el coeficiente de Okun en torno a -0.50 .

\section{Discusión}

¿Por qué el análisis refleja una disminución progresiva en el impacto del producto sobre el desempleo? Una posible respuesta se encuentra en la capacidad de la economía de generar empleos. Las figuras $8 \mathrm{a}$ y $8 \mathrm{~b}$ muestran la tasa de ocupación para dos períodos: 1961-1984 y 1990-2013. Para el primer período la medida incluye los empleados en relación a la población económicamente activa (PEA) -la oferta de trabajo en la economía. El segundo período calcula los empleados como proporción de la población en edad de trabajar (PET), la cual incluye la PEA y aquellos ciudadanos en edad de trabajar pero que no están activamente en el mercado de trabajo. Las cifras en las dos gráficas no son comparables pero pueden servir de base para iluminar vías que conduzcan al mejor entendimiento de los valores estimados para el coeficiente de Okun. 
Producto, desempleo y la Ley de Okun en la República Dominicana

Figura N. ${ }^{\circ} 8 \mathrm{a}$

Empleo como proporción de la población económicamente activa $(P E A)(\%)$

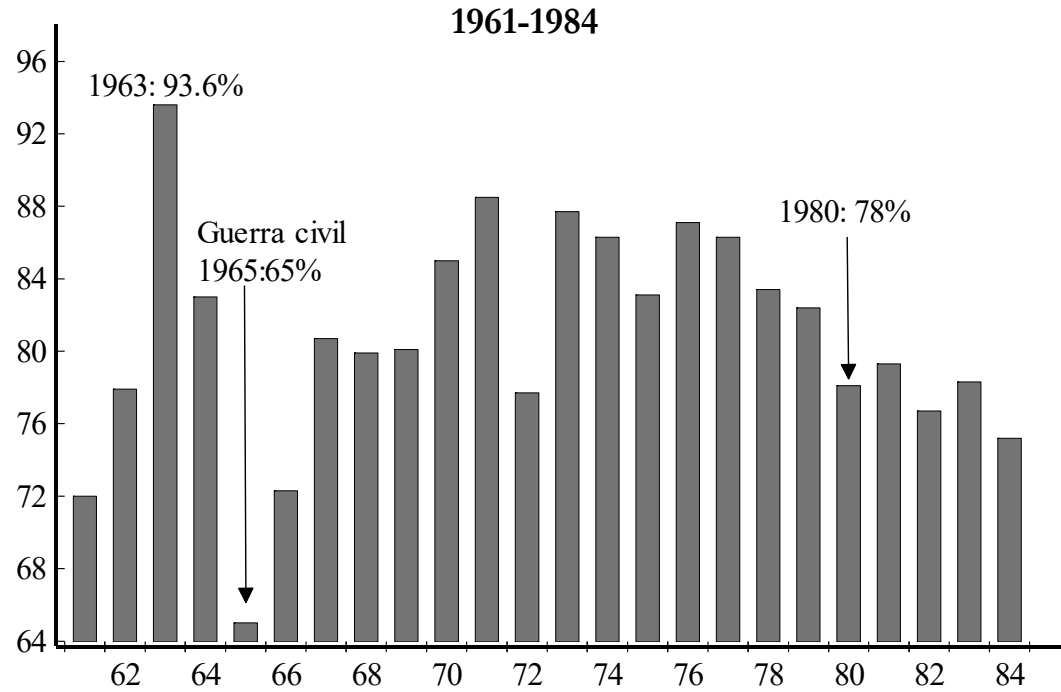

Fuente: Banco Central de la República Dominicana.

Figura $N .^{\circ} 8 b$

Empleo como proporción de la población en edad de trabajar (PET) (\%)

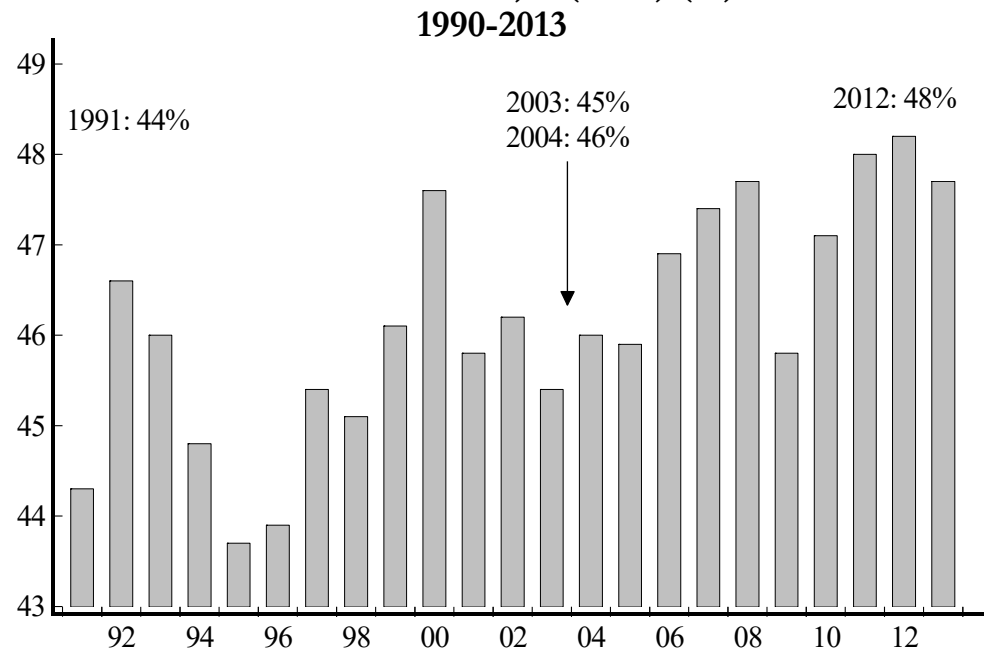

Fuente: Banco Central de la República Dominicana. 
La figura $\mathrm{N} .^{\circ} 8 \mathrm{a}$, la cual considera la oferta de trabajo, muestra que la tasa de ocupación alcanzó 93\% en 1963 y luego sufre una caída estrepitosa que la coloca en 65\% para el año 1965 como consecuencia de la Guerra civil. Subsecuentemente la tasa de ocupación fluctúa entre $76 \%$ y $88 \%$ hasta principios de la década del ochenta. La figura N. ${ }^{\circ} 8 \mathrm{~b}$, basada en datos que usan como referencia la PET, refleja una tasa de ocupación estable durante los años del noventa y los primeros años a partir del 2000. El valor más bajo es $44 \%$ y sucede un año después de la crisis del principio de la década. Y el valor más elevado es $48 \%$ en 2012. El 2012 fue un año de elecciones presidenciales y el gasto corriente del Gobierno en salarios alcanzó cifras relativamente elevadas, lo cual probablemente es un factor determinante del alto nivel de empleo. Los años 2003 y 2004, correspondientes a la crisis bancaria que costó al país alrededor de $20 \%$ del producto interno bruto, no registran caídas significativas en el empleo.

La economía creció razonablemente durante la década del noventa y principios del 2000, hasta el punto de llamar la atención con relación al crecimiento de otros países en América Latina (p. ej., Ocampo, 2004; Pozo, Sánchez-Fung y Santos-Paulino, 2013). Pero las cifras del empleo muestran un virtual estancamiento durante el período. La tendencia quizás refleja la existencia de problemas en la tasa de participación que pueden crear un límite sobre la oferta de trabajo. Para estudiar el problema recordemos que podemos escribir la población en edad de trabajar (PET), o fuerza de trabajo, de la siguiente manera

$$
P E T=\underbrace{E+U}_{\begin{array}{c}
P E A \\
\text { oferta de trabajo }
\end{array}}+F O T
$$

En la ecuación $11 E$ es el empleo y $U$ el desempleo, y estas variables forman la oferta de trabajo o PEA; FOT son los agentes económicos en edad de trabajar pero fuera de la oferta de trabajo. La variable FOT puede ser calculada en base a la ecuación 11. 


$$
F O T=P E T-\left\{\begin{array}{c}
\underbrace{E+U}_{\begin{array}{c}
P E A \\
\text { oferta de trabajo }
\end{array}} \\
\text { of }
\end{array}\right\}
$$

La variable FOT en la ecuación 12 puede estar explicada por muchos factores incluyendo problemas de incorporación a la oferta de trabajo por falta de destrezas o educación formal y períodos de desempleo que traumatizan al trabajador y lo conducen a salir permanentemente de la oferta de trabajo (p. ej., Clark, Georgellis y Sanfey, 2001). La figura N. 9 muestra la FOT calculada usando la ecuación 12 para el período 1991-2013 y datos anuales producidos por el Banco Central de la República Dominicana empleando la Encuesta Nacional de Fuerza de Trabajo. El patrón de comportamiento de FOT es estable y la variable es estacionaria de acuerdo a la prueba Dickey-Fuller aumentada que rechaza la hipótesis de una raíz unitaria al 1\% de probabilidad $\{-4.38(0.01)\}$. El valor promedio de FOT en la figura N. 9 registra $37 \%$ durante las últimas dos décadas, con máximo de $40 \%$ y mínimo de $33 \%$.

\section{Figura N. ${ }^{\circ} 9$}

Agentes fuera de la fuerza de trabajo, FOT (\%), 1991-2013

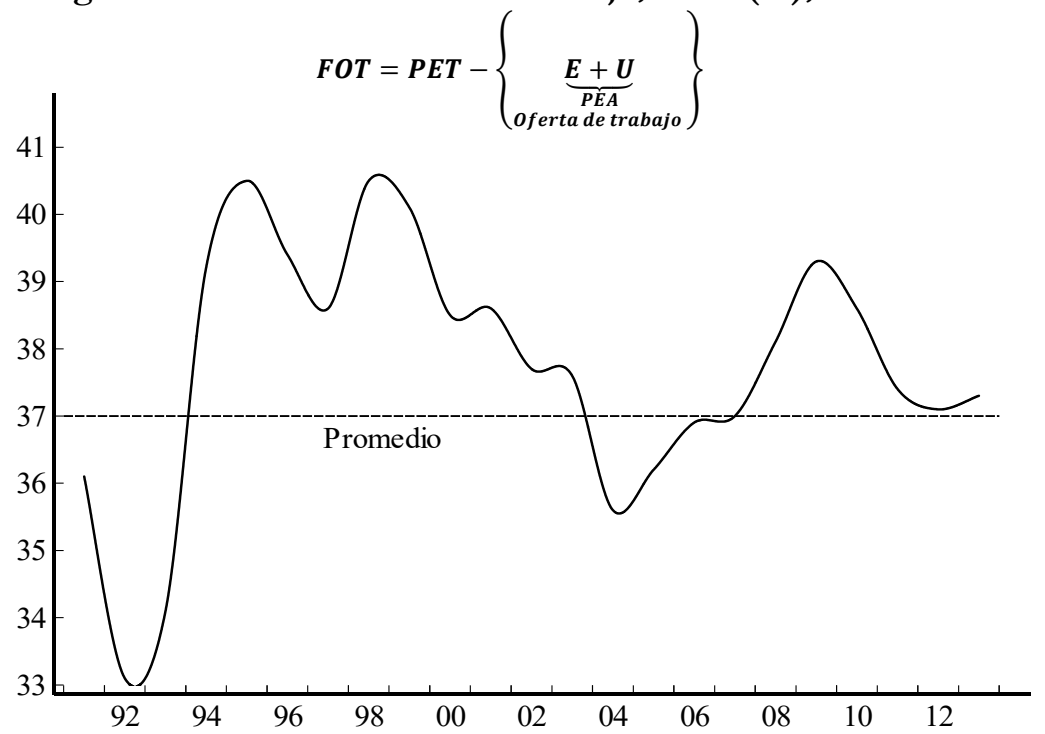

Fuente: Banco Central de la República Dominicana y cálculos del autor. 
¿En vista de las reformas estructurales aplicadas en los sectores educación, electricidad, salud, entre otros, y el correspondiente notable crecimiento económico, al menos en comparación con el resto de América Latina, por qué la variable FOT ha sido tan estable, arropando más de $1 / 3$ de la población en edad de trabajar durante las últimas dos décadas? El desempleo informal puede ser una explicación pero no existen cifras precisas sobre el sector. Como el artículo menciona anteriormente, problemas de falta de educación formal o destrezas, informalidad, así como el trauma por desempleo prolongado pueden ser algunos de los factores determinantes del volumen de agentes económicos catalogados como fuera de la fuerza de trabajo en la República Dominicana.

\section{Conclusión}

El trabajo investiga la relación entre el producto real y el desempleo en la República Dominicana empleando la Ley de Okun y series temporales sobre el producto y el desempleo. La modelación empírica revela que existe una relación de Okun estadísticamente significativa durante el último medio siglo pero el valor del coeficiente que conecta el producto con el desempleo ha caído progresivamente de acuerdo a las estimaciones recursivas. $\mathrm{Y}$ esa tendencia ha estado acompañada de una disminución en el producto real potencial de la economía implícito en las estimaciones econométricas. El artículo conduce al planteamiento de preguntas de potencial interés para investigaciones futuras.

Es particularmente importante tratar de entender por qué la cantidad de agentes económicos fuera de la oferta de trabajo ha fluctuado alrededor de 37\% durante las últimas dos décadas. Esa línea de investigación podría arrojar luz sobre debates acerca de la economía de la República Dominicana que surgen periódicamente como resultado de los episodios de crecimiento económico sin un correspondiente aumento en la cantidad y calidad del empleo y el bienestar de la población. El problema es conocido en la literatura 
como "crecimiento sin empleo". El tema no es nuevo para la República Dominicana o para otros países; ver, p. ej., Krueger et al. (2014) sobre los Estados Unidos, y Gregory (1997), Arnold Harberger (1997) y las discusiones y citas de muchos otros autores en Sánchez-Fung (2000). Las estimaciones en este trabajo arrojan luz sobre la relación entre el producto y desempleo analizando medio siglo de estadísticas e invitan a continuar indagando sobre el tema.

\section{Referencias}

Ball, L., Leigh, D., Furceri, D. \& Loungani, P. (2013). Okun's Law: Fit at fifty? NBER Working Paper No. 18668, Cambridge, MA.

Banco Central de la República Dominicana. Datos económicos. Recuperado de www.bancentral.gov.do

Clark, A. E., Georgellis, Y. \& Sanfey, P. (2001) Scarring: The psychological impact of past unemployment, Economica, 68, $221-41$.

Daly, M. C., Fernald, J., Jordà, O. \& Nechio, F. (2014). Interpreting deviations from Okun's Law, Federal Reserve Bank of San Francisco, Economic Letter, 2014-2012.

García Obregón, J. S. (2013). Una aplicación de la Ley de Okun para la República Dominicana, Oeconomía, 7(4), 2-6.

Gordon, R. J. (1984) Unemployment and potential output in the 1980s, Brookings Papers on Economic Activity, 2, 537-564.

Gregory, P. (1997). Empleo y desempleo en la República Dominicana. [Mimeo]. Santo Domingo, Banco Central de la República Dominicana.

Harberger, A. C. (1997). Economic policy and performance in the Dominican Republic. [Mimeo]. Santo Domingo. 
Knotek, E. S. (2007). How useful is Okun's Law? Federal Reserve Bank of Kansas City Economic Review, 4, 73-103.

Krueger, A. B., Cramer, J. \& Cho, D. (2014). Are the long-rerm unemployed on the margins of the labor market? Brookings Papers on Economic Activity. Recuperado de http://www.brookings.edu/ 22FBD006-AC39-40C1-BE16-D5142D070CD0/FinalDown load/DownloadId-644D87F4DE83293DD8C62951F6E40 170/22FBD006-AC39-40C1-BE16-D5142D070CD0/ / media/Projects/BPEA/Spring\%202014/2014a_Krueger.pdf

Ocampo, J. A. (2004). Latin America's growth and equity frustrations during structural reforms, Journal of Economic Perspectives, 18, 67-88.

Okun, A. M. (1962) Potential GNP: Its measurement and significance, American Statistical Association, Proceedings of the Business and Economics Statistics Section, 98-104.

Pozo, S., Sánchez-Fung, J. R. \& Santos-Paulino, A. U. (2013) Economic development strategies in the Dominican Republic, Chapter 18 in Augustin K. Fosu (Ed.) Achieving development success: Strategies and lessons from the developing world (pp. 383405). Oxford: Oxford University Press.

Prachwony, M. F. J. (1993). Okun's Law: Theoretical foundations and revisited estimates, Review of Economics and Statistics, 75, 331-336.

Sánchez-Fung, J. R. (2000). Employment and labour markets in the Dominican Republic: an overview of the literature, CEPAL Review, 71, 157-169.

Sánchez-Fung, J. R. (2005). Exchange rates, monetary policy, and interest rates in the Dominican Republic during the 1990s boom and new millennium crisis, Journal of Latin American Studies, 37, 727-738. 
World Bank World Development. (2014). Indicators. Recuperado de www.worldbank.org

\section{José R. Sánchez-Fung}

Es profesor de Economía en la Universidad de Nottingham, en Ningbo, China. También es Asociado de la Universidad de Londres, ILAS, en Inglaterra, Reino Unido, y miembro del Consejo Asesor Internacional de Ciencia y Sociedad, INTEC. Se ha desempeñado como economista visitante en varias universidades, incluyendo Columbia y Georgetown en los Estados Unidos, y Oxford en Inglaterra. El Dr. Sánchez-Fung también ha sido director del Departamento de Investigación Económica y Programación Monetaria en el Banco Central de la República Dominicana e investigador visitante en el Banco Central de Finlandia, en Helsinki. Sus intereses de investigación se encuentran en el amplio campo de la macroeconomía, con especial referencia sobre cuestiones empíricas y de relevancia para la política económica, y ha publicado artículos sobre la materia en revistas profesionales. Sánchez-Fung obtuvo los grados de Ph. D. y MA en Economía en la Universidad de Kent, en Inglaterra y la licenciatura en Economía en la PUCMM-RSTA, República Dominicana. 
